\title{
Degrees of Freedom of Wireless Networks - What a Difference Delay Makes
}

\author{
Viveck R. Cadambe, Syed A. Jafar \\ Electrical Engineering and Computer Science \\ University of California Irvine, \\ Irvine, California, 92697, USA \\ Email: vcadambe@uci.edu, syed@uci.edu
}

\begin{abstract}
We explore the impact of propagation delay on the degrees of freedom of wireless interference networks. For $K>2$ user interference channel we show through an example that propagation delays can increase the degrees of freedom by upto a factor of $K / 2$. We provide an example of node placement for a 4 user interference network such that the propagation delays for line-ofsight communication allow perfect interference alignment. We show that even if nodes are randomly placed, one can almost surely achieve sufficient interference alignment to approach the upperbound on the degrees of freedom by choosing the basic symbol duration small enough. An analogy with deterministic channel models is pointed out as an interesting mechanism to translate propagation delay based interference alignment schemes to delay-free Gaussian channel models.
\end{abstract}

\section{INTRODUCTION}

Interference is believed to be the principal limitation to the performance of wireless networks. In order to understand the role of interference, a promising approach is to de-emphasize the local additive noise at the nodes in relation to the signal and interference strength [1]. This is the philosophy behind deterministic channel models [1] and the degrees of freedom [2] characterizations, both of which provide valuable insights into the capacity of wireless networks. The classical problem in information theory that explores the nature of interference is the interference channel model [3]. The Gaussian interference channel capacity remained open for over thirty years, until a capacity approximation accurate to within one bit was found recently for the two user case [4]. The classical Gaussian interference channel problem ignores propagation delays as the signals travel from the transmitters to the receivers. If all paths to the same receiver suffer the same propagation delay then there is no loss of generality in this assumption as the delays merely imply a time shift at the receiver. However, in practice, each transmitter-receiver pair will be associated with a unique propagation distance, and therefore a unique propagation delay. Since propagation delay is an unavoidable physical reality in wireless communication, it is not clear to what extent the capacity results based on delay-free propagation models will be indicative of the capacity of wireless networks with propagation delays. It is this question that we explore in this paper. The metric that we use to estimate the capacity of wireless networks is the "degrees of freedom" $D$ [5]- which is defined as the ratio of the sum capacity $C(P)$ of the network to the $\log$ of the total transmit power $P$, in the limit that $P \rightarrow \infty$, where the local noise at each node is normalized to have unit variance.

$$
D=\lim _{P \rightarrow \infty} \frac{C(P)}{\log (P)}
$$

It is clear that degrees of freedom provide a capacity approximation $C(P)=D \log (P)+o(\log (P))$ whose accuracy approaches $100 \%$ as the total transmit power $P$ approaches infinity.

\section{A. System Model}

Consider a $K$ user interference network, i.e., an interference channel with $K$ transmitters and $K$ receivers. The signal input-output equations are:

$$
Y^{[i]}(n)=\sum_{j=1}^{n} H^{[i j]} X^{[j]}\left(n-d^{[i j]}\right)+Z^{[i]}(n)
$$

where at discrete time index $n, Y^{[i]}(n)$ is the received signal and $Z^{[i]}(n)$ the additive noise at the $i^{t h}$ receiver. $X^{[j]}(n)$ is the signal transmitted by the $j^{t h}$ transmitter and $H^{[i j]}$ is the channel coefficient and $n_{i, j}$ is the propagation delay between between transmitter $j$ and receiver $i$. Note that this model assumes propagation delays are integer multiples of the basic symbol duration. Non-integer delays will be addressed subsequently in this work. If we set all delays $d^{[i j]}$ equal to zero, we obtain the classical Gaussian interference channel model.

\section{THE IMPACT ON CAPACITY OF PROPAGATION DELAYS}

The first question that we address is whether propagation delay can significantly impact the capacity of a wireless network. Indeed, for the two user Gaussian 
interference channel, one can verify that neither the innerbounds nor the outerbounds of [4] are affected by the propagation delays. Since the inner and outerbounds are shown to be within 1 bit of each other, one can conclude that propagation delays do not impact the capacity of the 2 user Gaussian interference channel by more than 1 bit. This observation suggests that perhaps propagation delays do not significantly impact the capacity of the Gaussian interference channel. However, as we show next through an example, this is not the case for more than 2 users.

Consider a $K$ user interference channel where all channel coefficients are equal to one and all propagation delays are equal to zero, i.e. $H^{[i, j]}=1, n_{i, j}=0 \forall i, j \in$ $\{1,2, \cdots, K\}$. On this interference channel, all receivers observe statistically equivalent signals. Therefore, if a message can be decoded by any receiver, it can be decoded by all receivers. Consequently, the sum capacity of this interference channel is the sum capacity of the multiple access channel from all transmitters to, say, receiver 1 . This multiple access channel has only 1 degree of freedom, and therefore, the sum capacity of the interference channel is $\log (P)+o(\log (P))$.

Now, suppose with the same channel coefficients as before, we allow non-zero propagation delays. In particular, we assume:

$$
\begin{aligned}
& d^{[i j]} \bmod 2=0, \quad i=j \\
& d^{[i j]} \bmod 2=1, \quad i \neq j
\end{aligned}
$$

Thus, all desired signals arrive with an even propagation delay and all interfering signals arrive with an odd propagation delay at each receiver. On this interference channel, suppose all transmitters tranmit only over even time slots and are silent over odd time slots. Then, each receiver is able to hear his desired signals free of interference over the even time slots and all the interference is aligned over the odd time slots. Thus, even though all the channel coefficients are equal, this interference channel achieves a sum capacity of $K / 2 \log (P)+o(\log (P))$. We see through this example, that the presence of propagation delays can increase the degrees of freedom, and therefore the high SNR capacity by a factor of $K / 2$ for the $K$ user interference channel.

\section{Node Placement For INTERfERENCE ALIGNMENT}

The propagation delay example in the last section shows the capacity benefits of interference alignment. Since propagation delays correspond to propagation distances, the choice of propagation delays has clear implications for node placement. An interesting question is whether one can place nodes optimally in a way to

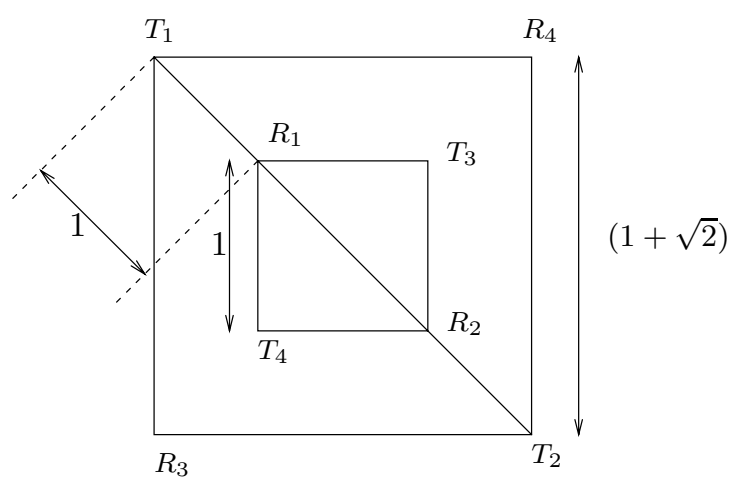

Fig. 1. A 4 user interference channel having 2 degrees of freedom

ensure interference alignment. If we allow only line-ofsight communication, the node placement directly determines the propagation delays. We present an example next to show how one may place 4 transmitters and their corresponding receivers such that every user is able to communicate free from interference for half the time.

Consider a 4 user interference channel. Let $d^{[j i]}$ indicate the delay between transmitter $i$ and receiver $j$. Let us place the nodes as in figure 1 and define $D_{1}=1$, $D_{2}=1+\sqrt{2}$. With this arrangement, the delays $d^{[i j]}$ satisfy the following conditions.

$$
\begin{gathered}
d^{[l 1]}=d^{[k 2]}=D_{1}, \quad l \neq 1, k \neq 2 \\
d^{[l 3]}=d^{[k 4]}=D_{2} \quad, l \neq 3, k \neq 4 \\
d^{[11]}=d^{[22]}=D_{2} \\
d^{[33]}=d^{[44]}=D_{1} \\
D_{1} \neq D_{2}
\end{gathered}
$$

We make time slotted, with each slot of length $\Delta=$ $D_{2}-D_{1}$. The transmission strategy is as follows. (See Fig. ??)

- Transmitters 1 and 2 transmit in even time slots and remain silent in odd time slots, i.e., they transmit between times duration $2 k \Delta$ and $(2 k+1) \Delta$

- Transmitters 3 and 4 transmit in odd time slots and remain silent in even time slots

Now with this transmitting strategy, it can be observed that all interference is perfectly aligned at all receivers. For example, at receiver 1 , interference from transmitters 3 and 4 arrive between times $(2 k-1) \Delta+D_{2}$ and $2 k \Delta+$ $D_{2}$, for all $k$. Also, since $2 k \Delta+D_{1}=(2 k-1) \Delta+D_{2}$, the interference from transmitter 2 at receiver 1 also arrives exactly between $(2 k-1) \Delta+D_{2}$ and $2 k \Delta+D_{2}$. Receiver 1 receives the desired signal from transmitter 1 between $2 k \Delta+D_{2}$ and $(2 k+1) \Delta+D_{2}$. Thus the desired 


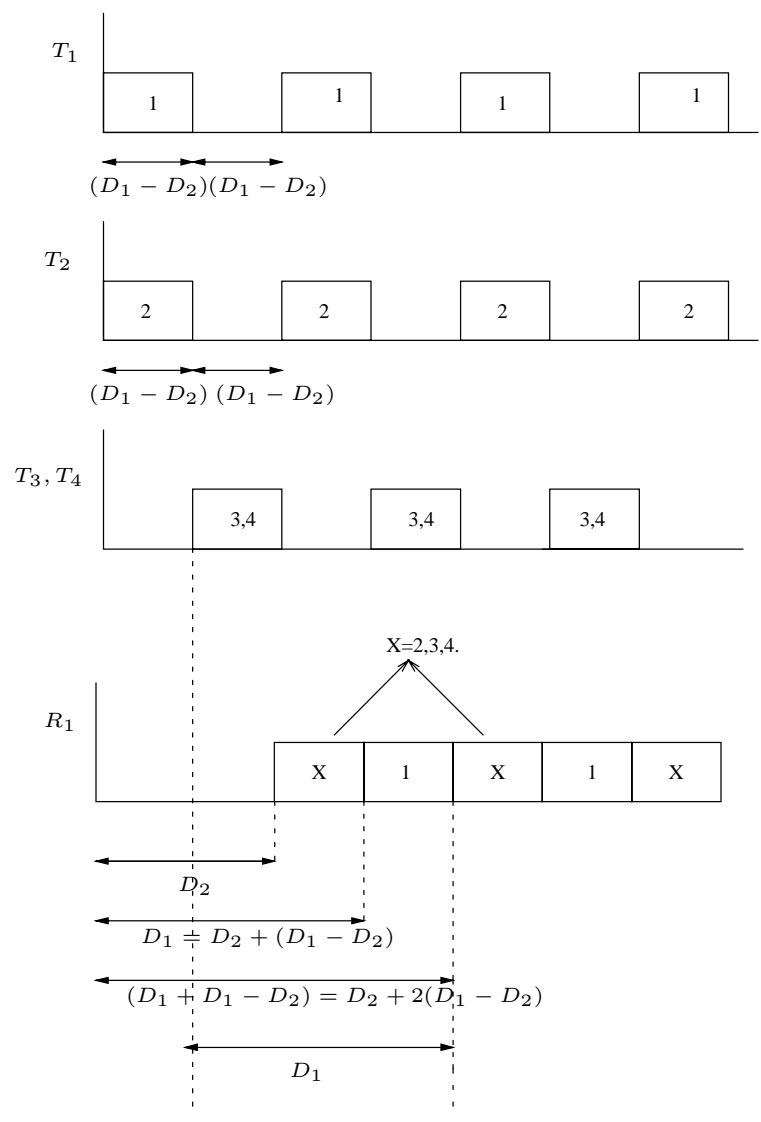

Fig. 2. Transmitted and received signals in the 4 user interference channel

signal at receiver 1 is shielded from all interference. Similarly, it can be verified that all receivers receive their desired signals free of interference so that the receive strategy is described as follows

- Receivers 1 and 2 listen between times $2 k \Delta+D_{2}$ and $(2 k+1) \Delta+D_{2}$ and ignore the received signal at other times.

- Receivers 3 and 4 listen between times $(2 k-1) \Delta+$ $D_{1}$ and $2 k \Delta+D_{1}$ and ignore the received signal at other times.

Since all the 4 'conversations' are active half the time, the channel has 2 degrees of freedom.

\section{RANDOM DELAYS}

In the previous section we explored node placement for interference alignment. The preceding example leads to a natural question - how much interference alignment is possible if the nodes are randomly placed. In this section we answer this question from a mathematical standpoint.
Theorem 1: The $K$ user interference channel with delays $d^{[i j]}$ independent of each other and uniformly distributed between a non-negative minimum and a finite maximum value has $K / 2$ degrees of freedom with probability 1 .

Proof: We show that $K / 2(1-\epsilon)$ degrees of freedom are achievable over this channel for any $\epsilon>0$ through an achievable scheme. For ease of exposition, let all delays have a uniform distribution between $D_{\min }$ seconds and $D_{\max }$ seconds. (Note that the result can easily be extended to the case where different delays have different uniform distributions as well) Now, we normalize the unit of time so that 1 unit of time is equal to $D_{\max }-D_{\min }$ seconds. Let $T_{\text {offset }}=\frac{D_{\min }}{D_{\max }-D_{\min }}$. Therefore, all delays are uniformly distributed between $T_{\text {offset }}$ and $T_{\text {offset }}+1$ units of time. Now we can write $d^{[i j]}-T_{\text {offset }}$ in its decimal expansion as

$$
d^{[i j]}-T_{\text {offset }}=0 . x_{1}^{[i j]} x_{2}^{[i j]} x_{3}^{[i j]} \ldots
$$

Since $d^{[i j]}-T_{\text {offset }}$ is uniformly distributed between 0 and 1 , elements of the sequence $x_{1}^{[i j]}, x_{2}^{[i j]} \ldots$ are i.i.d. Also, $x_{k}^{[i j]}$ can take any value from $\{0,1,2 \ldots 9\}$ with equal probability (of 0.1 ). We aim to achieve $K / 2(1-\epsilon$ ) degrees of freedom for any positive $\epsilon$. Now, choose $N$ so that $10^{-N}<\epsilon$. We wish to choose $n$ so that the set of random variables $\mathcal{M}_{n}=\left\{x_{l}^{[i j]}: i, j=1,2, \ldots K, l=\right.$ $n, n+1, \ldots n+N\}$ satisfy the following conditions.

- $x_{n}^{[i j]}$ is even if $i=j$ and odd if $i \neq j$

- $x_{n+1}^{[i j]}, x_{n+2}^{[i j]} \ldots x_{n+N}^{[i j]}$ are all equal to 0 for any $i, j=1,2 \ldots K$

Now, observe that, for a given $k$, the probability that the set of variables $\mathcal{M}_{k}$ satisfy the desired conditions is non-zero. Furthermore, the random variables of $\mathcal{M}_{k}$ are independent of the random variables in $\mathcal{M}_{k+N}$. Since there are infinite independent realizations of the set $\mathcal{M}_{k}$, with probability $1, \exists n \in \mathbb{N}$ such that the set $\mathcal{M}_{n}$ satisfies the desired conditions. In other words, by picking $n$ appropriately large, we can ensure that $\mathcal{M}_{n}$ satisfies the desired conditions. Now, given such $n$, let $T_{\text {slot }}=10^{-n}$ units of time. Also, let a symbol be of duration $T_{\text {sym }}=10^{-n}(1-\epsilon)$ units of time. The following communication strategy achieves $\frac{K}{2}(1-\epsilon)$ degrees of freedom

- All transmitters transmit a symbol at the beginning of every even time slot i.e. at times $2 n T_{\text {slot }}$ units

- Receiver $i$ listens between $d^{[i i]}+2 n T_{\text {slot }}$ units and $d^{[i i]}+(2 n) T_{\text {slot }}+T_{\text {sym }}$ units and ignore the received signal at other times

It can be easily verified that all the interference at receiver $i$ arrives between timeslots $d^{[i i]}+(2 n-1) T_{\text {slot }}-$ $T_{\text {slot }} \epsilon$ and $d^{[i i]}+(2 n) T_{\text {slot }}$ so that the desired signal is 
shielded from interference. Intuitively, the slot duration is chosen such that all desired delays are even and undesired delays are odd, within an error of $\epsilon$. To compensate for this error, the symbol duration is kept smaller than the slot duration by a factor of $\epsilon$. Thus, by picking $n$ appropriately large (or equivalently the symbol duration appropriately small), each 'conversation' achieves $1-\epsilon$ degrees of freedom so that $\frac{K}{2}(1-\epsilon)$ degrees of freedom are achieved in the entire system.

\section{DISCUSSION}

In the previous section, we established mathematically that even if nodes are randomly placed, one can almost surely achieve arbitrarily close to perfect interference alignment by choosing the basic symbol duration small enough. However, the mathematical argument presented above does not translate well into physics. For example, the argument relies on the following assumptions:

1) Symbol duration can be made arbitrarily small.

2) Delays can be measured to infinite precision.

The first assumption implies access to arbitrarily large bandwidth. Note that the degrees of freedom are normalized by the bandwidth, so that the above argument does not waste bandwidth from a mathematical standpoint. However, the symbol duration required to satisfy even a moderately small $\epsilon$ for even a moderate number $K$ of users quickly becomes too small to be physically feasible. The measurement of delays to an infinite precision is also precluded by physics. Thus, from a physical and a practical standpoint, the question remains open how much interference alignment is possible if nodes are randomly placed?

From a practical standpoint, the node placement example is quite relevant. Propagation delays have traditionally not been considered important because the speed of light is very high and the propagation delays are therefore often negligible. However, as we pursue higher data rates the basic symbol duration becomes smaller and the propagation delays become closer to the symbol duration. For example if the symbol duration is 1 microsecond, then a propagation distance of 300 meters corresponds to propagation delay of 1 symbol duration. Smaller symbol durations will make this distance even smaller. Even in current CDMA systems, the RAKE receiver is able to collect energy from many paths because the delay spread is larger than a basic chip duration. Thus, it is conceivable that propagation delays may be used for interference alignment. Another consideration is multipath. In the node placement example we assumed only line-of-sight propagation. The simple node placement solution will clearly run into problems when there is multi-path present. For this reason it seems that if node placement approach is to have any practical significance it might be in the scenario where very high carrier frequencies are used. It is well known that the signal attenuation is quite severe at very high frequencies so that only line of sight communication may be possible. Thus, one can imagine a wireless backbone of access points or extension points that are placed in a manner that facilitates interference alignment. The wireless backbone may use very high frequency carrier signals for the backhaul communication while the connection to the end users is made over a different frequency band.

From a theoretical perspective, the propagation delay construction for perfect interference alignment is quite interesting as well. It is shown in [?] that there is a clear analogy between the propagation delays and the deterministic channel model. In the deterministic channel model [6], the nodes transmit "bits" from a finite field and the channel raises some of the most significant bits above the noise floor while the remaining bits are lost. It turns out that the channel shifting the bits on the deterministic channel is quite similar to the propagation delays which shift the signals in time. Thus, interference alignment schemes based on propagation delays can be equivalently viewed as interference alignment schemes on the deterministic channel model of the same network without propagation delays. It has been shown in [?] that the interference alignment scheme on the deterministic channel model can be directly translated into the classical Gaussian channel model with no propagation delays as well. This is especially relevant because interference alignment schemes are in general not known for channels with constant coefficients and no propagation delays, when each node has only one antenna.

Finally, propagation delay based interference alignment schemes can be constructed for wireless networks besides the interference channels. For example, consider a two user $X$ network, i.e., a network of two transmitters and two receivers where each transmitter has an independent message for each receiver, so that the network has a total of 4 messages. The outerbound on the degrees of freedom for this network is shown to be equal to $4 / 3$ in [2]. In the delay-free case, the outerbound is also shown to be achievable if the channel coefficients vary in time or frequency. However, if the channel coefficients are constant then it is not known if perfect interference alignment is possible. Now, suppose we allow propagation delays, so that the signals from transmitter 1 suffer a propagation delay of one symbol while the signals from transmitter 2 suffer a propagation delay of 2 symbols to receiver 1 and 3 symbols to receiver 2 . Then, by carefully interleaving the two messages at each transmitter over 
three time slots one can create a perfect interference alignment scheme that achieves the outerbound of $4 / 3$ degrees of freedom, as shown in [7]. The propagation delay example is translated into the deterministic channel model and then into the delay free Gaussian channel model to find an interference alignment scheme for the $X$ channel with constant coefficients and no delays.

It is not known in general if propagation delay examples can be constructed for every network. In particular, propagation delay based interference alignment examples are not known for $\mathrm{X}$ networks with more than 2 transmitters or receivers. Finding such examples is an interesting open problem. Another open problem is to find optimal node placements for perfect interference alignment on both the interference channel with more than 4 users as well as $X$ networks with multiple (more than 2) transmitters and receivers.

\section{REFERENCES}

[1] A. S. Avestimehr, S. Diggavi, and D. Tse, "Wireless network information flow," Sep 2007. Allerton Conference.

[2] S. Jafar and S. Shamai, "Degrees of freedom region for the MIMO X channel," in arXiv:cs.IT/0607099v3, May 2007.

[3] A. B. Carleial, "Interference channels," IEEE Trans. Inform. Theory, vol. 24, no. 1, pp. 60-70, 1978.

[4] R. Etkin, D. Tse, and H. Wang, "Gaussian interference channel capacity to within one bit," submitted to IEEE Transactions on Information Theory, Feb. 2007.

[5] L. Zheng and D. N. Tse, "Packing spheres in the Grassmann manifold: A geometric approach to the non-coherent multi-antenna channel,," IEEE Trans. Inform. Theory, vol. 48, pp. 359-383, Feb 2002.

[6] A. S. Avestimehr, S. Diggavi, and D. Tse, "A deterministic approach to wireless relay networks," Oct 2007, arXiv:cs.IT/0710.3777.

[7] S. Jafar, S. Shamai, and V. Cadambe, "Interference alignment on the deterministic channel and application to gaussian networks," Submitted to 2008 ISIT.

[8] V. Cadambe and S. Jafar, "Degrees of freedom of wireless X networks," in Preprint available through http://newport.eecs.uci.edu/ syed, 2007. 